\title{
Simple and Rapid Method for Determination of Abemaciclib in Human Serum Using Supported Liquid Extraction Pretreatment and LC-MS/MS Analysis
}

\author{
Naoto Sekizaki ${ }^{1,2}$, Hideaki Yashima1, Takuya Araki ${ }^{1,2 *}$, Koujirou Yamamoto ${ }^{1,2}$ \\ ${ }^{1}$ Department of Clinical Pharmacology and Therapeutics, Gunma University Graduate School of \\ Medicine, 3-39-22 Showa-machi, Maebashi 371-8511, Japan \\ ${ }^{2}$ Department of Pharmacy, Gunma University Hospital, 3-39-15 Showa-machi, Maebashi 371-8511, \\ Japan
}

Received : 01 Sept 2020, Revised : 04 Oct 2020, Accepted : 19 Nov 2020, Published : 5 Dec 2020

\begin{abstract}
We developed a simple and rapid method for the determination of abemaciclib in human serum using supported liquid extraction (SLE) method for pretreatment and LC-MS/MS. Abemaciclib was extracted using SLE method with methyltert-butyl ether (MTBE) as elution buffer, and analyzed by LC-QTOF MS system, LCMS9030 (Shimadzu). Abemaciclib and fluconazole (internal standard) were detected with ESI spray in positive ionization mode, and the transition were set at 507.3/393.1629 for abemaciclib and 307.1/220.0677 for fluconazole. The retention times of abemaciclib and fluconazole were 2.76 and $2.98 \mathrm{~min}$, respectively, and good linearity was obtained from $20-1,000 \mathrm{ng} / \mathrm{mL}$ for abemaciclib. The regression equation (weight $=1 / \mathrm{x} 2$ ) describing the calibration curve in human serum was $\mathrm{y}=0.0196 \mathrm{x}-0.056(\mathrm{R} 2=0.999)$, where $\mathrm{y}$ is the peak area ratio of abemaciclib against the IS and $\mathrm{x}$ is the nominal concentration of abemaciclib. The intraand inter-assay accuracy varied between $-4.3-1.7 \%$, and the precision varied between $0.90-$ $6.19 \%$. The mean recovery rate of abemaciclib was $87.7 \pm 4.3 \%$, and the mean matrix factor was $1.00 \pm 0.083$. Our method offers speed and simplicity of sample preparation, which is one of great advantages in the analysis of clinical specimens. We believe that the present method will contribute to establishing a methodology for determining the optimal dose of abemaciclib for individual patients.
\end{abstract}

Keywords: abemaciclib, SLE, supported liquid extraction, quantification

\section{Introduction}

Abemaciclib is an orally administered small molecule inhibitor of cyclin-dependent kinases (CDKs) 4 and 6 [1]. It has reported to be effective as a first-line treatment for hormone receptorpositive, HER2-negative advanced breast cancer when used in combination with a fulvestrant or nonsteroidal aromatase inhibitor [2-4]. On the other hand, hematological toxicity and diarrhea, common adverse drug reactions (ADRs) of abemaciclib, are known to results in drug intolerance in patients [5, 6]. Recently, some ADRs of abemaciclib have been reported to be dose dependent [7]. However, the pharmacokinetics of abemaciclib vary largely among individual patient [8] and have been reported to be affected by concomitant use of drugs such as CYP3A modulators [9]. So, to develop a methodology to determine the appropriate dose for each patient, establishment of method for quantifying abemaciclib is needed.

In 2015, Raub et al. detected abemaciclib and palbociclib using LC-MS/ MS in the study of exposure of CDK4 and 6 inhibitors to brain, but no further details of the methods and their performance were described [10]. Recently, 
Martinez-Chavez et al. also reported the quantitative method of abemaciclib, palbociclib, and ribociclib in human and mouse matrices using LC-MS/MS [11]. However, this method could not be applicable to the analysis of clinical specimens directly, because the measurement range of abemaciclib was set at $2-150 \mathrm{ng} / \mathrm{mL}$ in their study though the clinical concentration of abemaciclib in the blood is known to vary greatly and be about 50-300 $\mathrm{ng} / \mathrm{mL}$ [12]. In addition, the protein precipitation method used in the report is widely used for sample pretreatment because of simplicity and ease, however, it is also known to place a heavy load on an analytical system, especially MS system. Considering the analysis of a large number of clinical specimens, methods should be simple, easy, and has a low load on the detection system. Thus, a method of measurement of abemaciclib in clinical specimens is needed to establish safer and more effective therapy using abemaciclib.

Recently supported liquid extraction (SLE) method was developed as a new pretreatment method to replace the widely used liquid-liquid extraction (LLE) method, and have been already used for detection of some drugs and other substances in biological fluids [13-19]. SLE method is based on the same chemistry as LLE. However, instead of using a separation funnel and partitioning between two immiscible liquids the extraction is performed on a solid support made of diatomaceous earth. Compared to ordinary solid-phase extraction (SPE) or LLE, SLE has many advantages such as the absence of emulsion formation, high-extraction efficiency, simplicity of procedure, and short time required [14-23]. In addition, since SLE does not need the deproteinization which is needed for SPE as a pretreatment, SLE also has the advantage of avoiding coprecipitation with proteins associated with the deproteinization process [15].

In this study, to establish safer and more effective therapy using abemaciclib, we developed a method for the measurement of abemaciclib in human blood that can be applicable for clinical use using SLE method and LC-MS/MS analysis.

\section{Methods}

\subsection{Materials}

Abemaciclib and fluconazole was purchased from LKT Laboratories, Inc. (St. Paul, Minnesota, USA). Human serum pool (P/N. 12181201, lot\#. BJ14005A) was purchased from COSMO Bio Co., LTD (Tokyo, Japan). ISOLUTE SLE+ $400 \mu \mathrm{L}$ 96-well plate (SLE array plate) was purchased from Biotage Japan Ltd. (Tokyo, Japan). All other reagents were obtained from commercial sources and were LCMS or HPLC-grade or special-grade reagents.

\subsection{Preparation of stock solutions, working solutions, calibration samples, and quality control samples}

Primary stock solutions of $1.0 \mathrm{mg} / \mathrm{mL}$ of abemaciclib and fluconazole were separately prepared in ethanol and methanol, respectively. Fluconazole was used as an ISTD because it was found to be extracted by same pretreatment process, and show the similar elution behavior in LC separation as abemaciclib, and has been rarely used in combination with abemaciclib due to its CYP3A4 inhibitory effect. Primary stock solution of abemaciclib was diluted with the initial mobile phase $(0.1 \%$ formic acid in Milli-Q water:acetonitrile $=15: 85$ ) to yield standard working solutions $(0.1,0.25,0.5,1.0,2.5$, and 5.0 $\mu \mathrm{g} / \mathrm{mL})$. The ISTD was diluted in $1 \%$ aqueous ammonia to $50 \mathrm{ng} / \mathrm{mL}$. The stock solutions and other diluted solutions were stored at $-20^{\circ} \mathrm{C}$ and $4^{\circ} \mathrm{C}$, respectively, under dark conditions. All solutions were equilibrated to room temperature before use. Calibration samples and quality control (QC) samples were prepared by spiking blank serum with a given volume of different working solutions. The calibration samples consisted of six concentrations of abemaciclib: 20, 50, 100, 200, 500, and $1,000 \mathrm{ng} / \mathrm{mL}$ as concentrations in serum. QC samples of abemaciclib as low level (L), middle level (M), and high level $(\mathrm{H})$ were set at 20, 200, and 1,000 $\mathrm{ng} / \mathrm{mL}$, respectively.

\subsection{Sample preparation}

The serum sample was treated with SLE method. A mixture of $100 \mu \mathrm{L}$ of blank serum, 20 $\mu \mathrm{L}$ of standard solution in initial mobile phase, 
and $280 \mu \mathrm{L}$ of $1 \%$ aqueous ammonia containing $50 \mathrm{ng} / \mathrm{mL}$ of fluconazole was applied into an

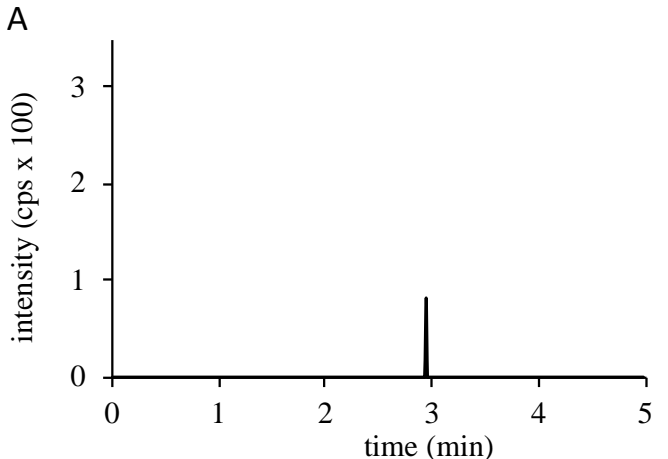

C
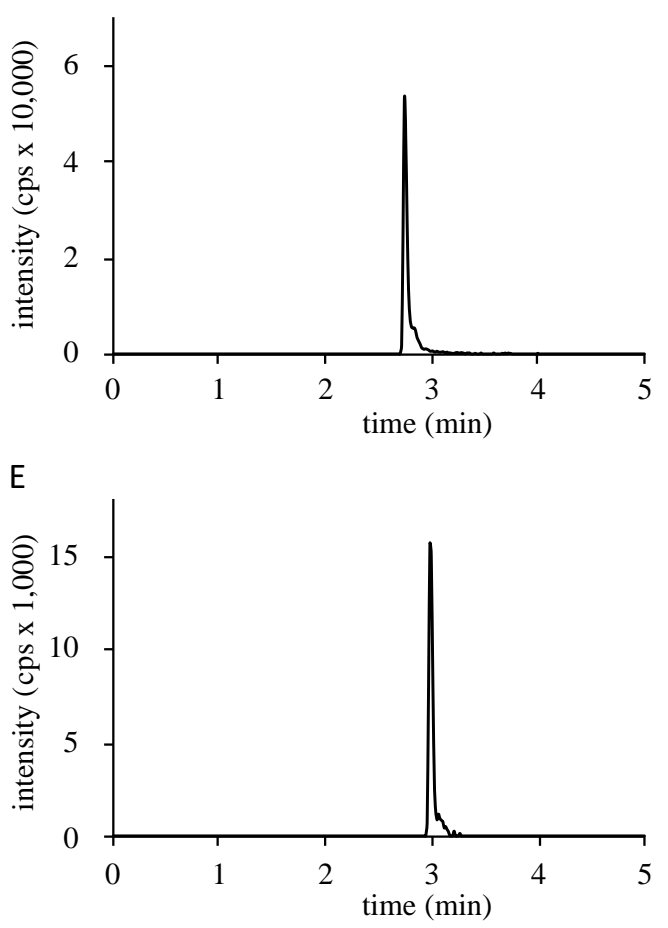

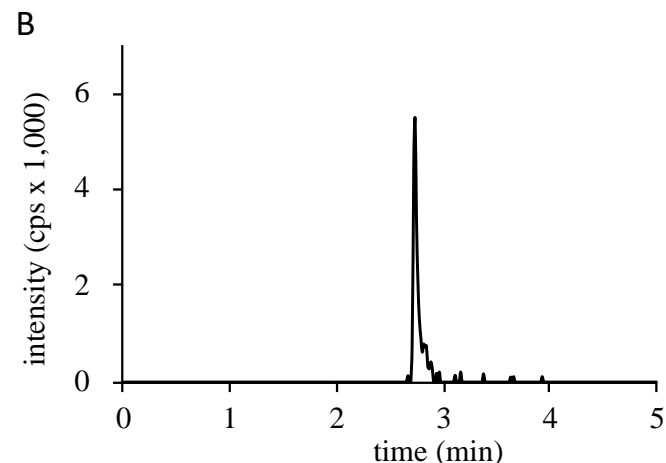

D

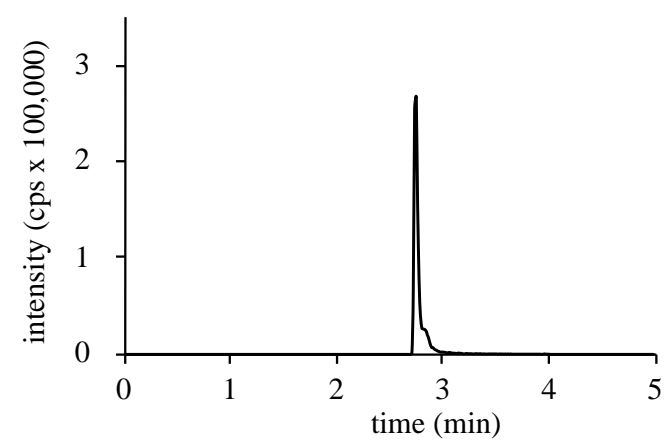

Figure 1. Typical chromatograms of abemaciclib and fluconazole in human serum

A D: Chromatograms of abemaciclib in blank serum (A), spiked with $20 \mathrm{ng} / \mathrm{mL}$ (B), spiked with 200 $\mathrm{ng} / \mathrm{mL}(\mathrm{C})$, and spiked with $1,000 \mathrm{ng} / \mathrm{mL}$ (D)

E: Chromatogram of fluconazole as ISTD in serum sample

ISOLUTE SLE $+400 \mu \mathrm{L}$ 96-well plate (SLE array plate) (Biotage) sitting on top of a clean 96-well collection plate. Positive pressure was applied for $2 \mathrm{sec}$ to initiate flow, and sample were allowed to stand for $5 \mathrm{~min}$ to absorb to the diatomaceous earth extraction bed. As elution buffer, $900 \mu \mathrm{L}$ of methyltert-butyl ether (MTBE) was added to the SLE array plate. After waiting for $5 \mathrm{~min}$, a further aliquot of MTBE $(900 \mu \mathrm{L})$ was applied and eluted within around $5 \mathrm{~min}$ by standing under natural gravity. The samples in the collection plate were evaporated to dryness under reduced pressure at room temperature. The residue was dissolved in 1 $\mathrm{mL}$ of initial mobile phase, and $2 \mu \mathrm{L}$ of samples were used for LC-MS/MS analysis.

\subsection{Detection of abemaciclib by LC-MS/MS}

Quadrupole time-of-flight chromatograph mass spectrometer was used to detect abemaciclib. LCMS 9030 (Shimadzu, Kyoto, Japan) with ESI spray in positive ionization mode was used with the following parameters: interface voltage, $1,000 \mathrm{~V}$; interface temperature, $300^{\circ} \mathrm{C}$; heat block temperature, $400^{\circ} \mathrm{C}$; desolvation line 
temperature, $250^{\circ} \mathrm{C}$; nebulizer gas $\left(\mathrm{N}_{2}\right)$ flow, 3 $\mathrm{L} / \mathrm{min}$; heating gas (air) flow, $10 \mathrm{~L} / \mathrm{min}$; drying gas $\left(\mathrm{N}_{2}\right)$ flow, $10 \mathrm{~L} / \mathrm{min}$; and collision-induceddissociation gas pressure, $230 \mathrm{kPa}$. The following transitions were monitored: 507.3/393.1629 for abemaciclib, and 307.1/220.0677 for fluconazole. Collision energies were $26.0 \mathrm{~V}$, and $16.0 \mathrm{~V}$, respectively. For separation analysis, LC was performed with a Nexera $\mathrm{X} 2^{\circledR}$ system (Shimadzu). The system consisted of LC-30AD as solvent systems, SIL-30AC as an auto sampler, CTO-20AC as a column heater, and CBM-20A as a system controller. An ACQUITY UPLC ${ }^{\circledR}$ BEH130 C18 column $(2.1 \mathrm{~mm} \times 100 \mathrm{~mm}, 1.7$ $\mu \mathrm{m})$ (Waters, Milford, MA) was used as the LC column. The LC conditions were as follows: column temperature, $40^{\circ} \mathrm{C}$; mobile phase, $0.1 \%$ formic acid in Milli-Q water (A) and acetonitrile (B); flow rate, $0.3 \mathrm{~mL} / \mathrm{min}$; and gradient program, 15 to $35 \% \mathrm{~B}$ in $5 \mathrm{~min}, 35$ to $80 \% \mathrm{~B}$ in $1 \mathrm{~min}, 80 \% \mathrm{~B}$ in $1 \mathrm{~min}, 80$ to $15 \% \mathrm{~B}$ in $1 \mathrm{~min}$, and $15 \% \mathrm{~B}$ in $3 \mathrm{~min}$.

\subsection{Assay validation}

The method was validated according to guidance of ICH [23]. Precision was measured as the coefficient of variation expressed as a percentage, and accuracy was expressed as the relative error of the nominal versus the measured concentration. The intra-assay variability was tested by measuring three different serum samples against the same calibration curve. The inter-assay variability was tested on three different days and a new calibration curve was constructed for each day. The matrix effect was also evaluated quantitatively by measurement of the matrix factor.

\section{Results}

3.1 Detection of abemaciclib and fluconazole

The typical chromatograms obtained from blank pooled human serum (PHS) and from blank PHS spiked with abemaciclib (20, 200, 1,000 $\mathrm{ng} / \mathrm{mL}$ as concentrations in serum) and fluconazole (14 $\mathrm{ng} / \mathrm{mL}$ as concentration in serum) were shown in figure 1 . The retention times of abemaciclib and fluconazole were 2.76 and 2.98 min, respectively.

\subsection{Linearity, Accuracy and Precision}

Good linearity was obtained from 20-1,000

$\mathrm{ng} / \mathrm{mL}$ for abemaciclib. The regression equation (weight $=1 / \mathrm{x}^{2}$ ) describing the calibration curve in PHS was $y=0.0196 x-0.056\left(R^{2}=0.999\right)$, where $\mathrm{y}$ is the peak area ratio of abemaciclib against the IS and $\mathrm{x}$ is the nominal concentration of abemaciclib.

Accuracy and precision were shown in Table 1 . The results were within the accepted limits and therefore the assay was accurate and precise.

Table 1. Intra- and inter-assay validation

\begin{tabular}{cccc}
\hline $\begin{array}{c}\text { Nominal concentration } \\
(\mathrm{ng} / \mathrm{mL})\end{array}$ & $\begin{array}{c}\text { Measured concentration } \\
(\mathrm{ng} / \mathrm{mL})\end{array}$ & $\begin{array}{c}\text { Accuracy } \\
(\mathrm{RE} \%)\end{array}$ & $\begin{array}{c}\text { Precision } \\
(\mathrm{CV} \%)\end{array}$ \\
\hline Intraday-assay $(\mathrm{n}=3)$ & $20.3 \pm 0.31$ & 1.70 & 1.52 \\
20 & $48.0 \pm 1.59$ & -4.09 & 3.31 \\
50 & $96.9 \pm 1.99$ & -3.15 & 2.06 \\
100 & $196.5 \pm 2.41$ & -1.73 & 1.23 \\
200 & $485.6 \pm 16.9$ & -2.89 & 3.48 \\
500 & $976.9 \pm 26.2$ & -2.31 & 2.69 \\
1,000 & & & \\
Interday-assay $(\mathrm{n}=3)$ & & 1.28 & 6.19 \\
20 & $20.3 \pm 1.26$ & -4.30 & 2.96 \\
50 & $47.9 \pm 1.42$ & & \\
\hline
\end{tabular}




$\begin{array}{cccc}100 & 98.1 \pm 1.23 & -1.94 & 1.26 \\ 200 & 198.2 \pm 6.04 & -0.91 & 3.05 \\ 500 & 499.3 \pm 25.9 & -0.13 & 5.19 \\ 1,000 & 993.2 \pm 8.92 & -0.68 & 0.90\end{array}$

Data are presented as mean \pm standard deviation

\subsection{Extraction recovery}

The recovery of abemaciclib was acceptable and reproducible (Table 2). The mean recovery was $87.7 \%$.

Table 2. Recovery rate

\begin{tabular}{lr}
\hline Concentration & \multicolumn{1}{l}{ Recovery rate } \\
\hline$(\mathrm{ng} / \mathrm{mL})$ & $(\%)$ \\
20 & $86.0 \pm 2.54$ \\
200 & $88.2 \pm 4.49$ \\
1,000 & $88.8 \pm 6.27$ \\
\hline
\end{tabular}

Data are presented as mean \pm standard deviation

\subsection{Interference and Matrix effect}

The RSD was less than $15 \%$ and peak of abemaciclib was not detected from blank PHS sample. The average of matrix factor was $1.00 \pm$ 0.083 (Table 3), suggesting no significant matrix effect observed and no adverse impact on the quality of the data produced.

Table 3. Matrix efficacy

\begin{tabular}{ccl}
\hline $\begin{array}{l}\text { Concentration } \\
(\mathrm{ng} / \mathrm{mL})\end{array}$ & Matrix factor & $\begin{array}{l}\text { optim } \\
\text { Refe }\end{array}$ \\
\hline 20 & $0.976 \pm 0.014$ & 1. \\
200 & $1.02 \pm 0.034$ & \\
1,000 & $1.02 \pm 0.037$ &
\end{tabular}

Data are presented as mean \pm standard deviation

\section{Discussion and Conclusion}

We established a method for measurement of abemaciclib in human serum, which can be used directly for the analysis of clinical specimens, using SLE pretreatment method and LC/MS/MS analysis for the first time. In our method, SLE was used for pretreatment of serum sample. SLE has many advantages such as the high-extraction efficiency, simplicity of procedure, and short time required compared to
LLE or SPE [14-23]. In fact, with our method, the time required for pretreatment was less than 1 hour, a recovery rate of abemaciclib was around $90 \%$, and almost no matrix effect was found. These points are very advantageous as an analytical method used for measurement of drugs in clinical specimens. Furthermore, since our method needs simple steps using a column and simple steps, it is not easily affected by variations in the operator's skill. Now, this method has been used as clinical test in clinical setting, and the concentrations of abemaciclib in the blood of patients who received abemaciclib therapy were successfully measured.

In conclusion, we established a method for measurement of abemaciclib in human serum, which can be used directly for the analysis of clinical specimens, for the first time. Our method offers speed and simplicity of sample preparation, which is one of great advantages in the analysis of clinical specimens. We believe that the present method will contribute to establishing a methodology for determining the optimal dose for individual patients.

\section{References}

. Gelbert LM, Cai S, Lin X, SanchezMartinez C, Prado MD, Lallena MJ, et al. Preclinical characterization of the CDK4/6 inhibitor LY2835219: in-vivo cell cycle-dependent/independent antitumor activities alone/in combination with gemcitabine. Invest New Drugs. 2014;32(5):825-37.

2. Sledge GW, Toi M, Neven P, Sohn J, Inoue K, Pivot X, et al. MONARCH 2: Abemaciclib in Combination With Fulvestrant in Women With HR+/HER2Advanced Breast Cancer Who Had Progressed While Receiving Endocrine Therapy. J Clin Oncol. 2017;35(25):287584. 
3. Goetz MP, Toi M, Campone M, Sohn J, Paluch-Shimon S, Huober $J$, et al. MONARCH 3: Abemaciclib As Initial Therapy for Advanced Breast Cancer. J Clin Oncol. 2017;35(32):3638-46 (2017).

4. Johnston S, Martin M, Leo AD, Im S, Awada A, Forrester T, et al. MONARCH 3 final PFS: a randomized study of abemaciclib as initial therapy for advanced breast cancer. NPJ Breast Cancer. 2019;5:5

5. Kassem L, Shohdy KS, Lasheen S, AbdelRahman O, Bachelot T. Hematological adverse effects in patients with breast cancer treated with cyclin-dependent kinase 4 and 6 inhibitors: a systematic review and meta-analysis. Breast cancer. 2018;25(1):17-27

6. Ramos-Esquivel A, Hernandez-Steller H, Savard MF, Landaverde DU. Cyclindependent kinase 4/6 inhibitors as firstline treatment for post-menopausal metastatic hormone receptor-positive breast cancer patients: a systematic review and meta-analysis of phase III randomized clinical trials. Breast Cancer. 2018; 25(4):479-88.

7. Fujiwara Y, Tamura K, Kondo S, Tanabe Y, Iwasa S, Shimomura A, et al. Phase 1 study of abemaciclib, an inhibitor of CDK 4 and 6 , as a single agent for Japanese patients with advanced cancer. Cancer Chemother Pharmacol. 2016;78(2):281-8.

8. Tate SC, Sykes AK, Kulanthaivel P, Chan EM, Turner PK, Cronier DM. A Population Pharmacokinetic and Pharmacodynamic Analysis of Abemaciclib in a Phase I Clinical Trial in Cancer Patients. Clin Pharmacokinet. 2018;57(3):335-44.

9. Posada MM, Morse BL, Turner PK, Kulanthaivel P, Hall SD, Dickinson GL. Predicting Clinical Effects of CYP3A4 Modulators on Abemaciclib and Active Metabolites Exposure Using Physiologically Based Pharmacokinetic Modeling. J Clin Pharmacol. 2020;60(7):915-30.

10. Raub TJ, Wishart GN, Kulanthaivel P, Staton BA, Ajamie RT, Sawada GA, et al.
Brain Exposure of Two Selective Dual CDK4 and CDK6 Inhibitors and the Antitumor Activity of CDK4 and CDK6 Inhibition in Combination with Temozolomide in an Intracranial Glioblastoma Xenograft. Drug Metab Dispos. 2015;43(9):1360-71.

11. Martínez-Chávez A, Rosing H, Hillebrand M, Tibben M, Schinkel AH, Beijnen JH. Development and validation of a bioanalytical method for the quantification of the CDK4/6 inhibitors abemaciclib, palbociclib, and ribociclib in human and mouse matrices using liquid chromatography-tandem mass spectrometry. Anal Bioanal Chem. 2019;411:5331-45.

12. Maeda $A$, Irie $K$, Hashimoto $N$, Fukushima S, Ando H, Okada A, et al. Serum concentration of the CKD4/6 inhibitor abemaciclib, but not of creatinine, strongly predicts hematological adverse events in patients with breast cancer: a preliminary report. Invest New Drugs. 2020; doi: 10.1007/s10637-020-00994-3. Online ahead of print.

13. Moon JY, Lee HS, Kim JH, Lee JH, Choi MH. Supported liquid extraction coupled to gas chromatography-selective mass spectrometric scan modes for serum steroid profiling. Anal Chim Acta. 2018;1037:281-92.

14. Hawley JM, Owen LJ, Debono M, Newell-Price J, Keevil BG. Development of a rapid liquid chromatography tandem mass spectrometry method for the quantitation of serum dexamethasone and its clinical verification Ann Clin Biochem. 2018;55(6):665-72.

15. Kohira T, Kita Y, Tokuoka SM, Shiba M, Satake M, Shimizu T. Characterization of supported liquid extraction as a sample pretreatment method for eicosanoids and related metabolites in biological fluids. J Chromatgr B. 2019;1124:298-307.

16. Svanström C, Hansson GP, Svensson LD, Sennbro CJ. Development and validation of a method using supported liquid extraction for the simultaneous 
determination of midazolam and 1'hydroxy-midazolam in human plasma by liquid chromatography with tandem mass spectrometry detection. J Pharm Biomed Anal. 2012;58:71-7.

17. Rao RN, Prasad KG, Kumar KVS, Ramesh B. Diatomaceous earth supported liquid extraction and LC-MS/MS determination of elvitegravir and ritonavir in rat plasma: application to a pharmacokinetic study. Anal Methods. 2013;5:6693-9.

18. Rositano J, Harpas P, Kostakis C, Scott T. Supported liquid extraction (SLE) for the analysis of methylamphetamine, methylenedioxymethylamphetamine and delta-9-tetrahydrocannabinol in oral fluid and blood of drivers. Forensic Sci Int. 2016;265: 125-30.

19. Jiang H, Cao H, Zhang Y, Fast DM. Systematic evaluation of supported liquid extraction in reducing matrix effect and improving extraction efficiency in LCMS/MS based bioanalysis for 10 model pharmaceutical compounds. J Chromatogr B. 2012;891-892:71-80.

20. Hempen C, Elfering S, Mulder AHL, Bergh FAJTM, Maatman RGHJ. Dexamethasone suppression test: development of a method for simultaneous determination of cortisol and dexamethasone in human plasma by liquid chromatography/tandem mass spectrometry. Ann Clin Biochem. 2012;49:170-6.

21. Ray JA, Kushnir MM, Rockwood AL, Meikle AW. Analysis of cortisol, cortisone and dexamethasone in human serum using liquid chromatography tandem mass spectrometry and assessment of cortisol: cortisone ratios in patients with impaired kidney function. Clin Chim Acta. 2011;412:1221-8.

22. McWhinney BC, Briscoe SE, Ungerer JPJ, Pretorius CJ. Measurement of cortisol, cortisone, prednisolone, dexamethasone and 11-deoxycortisol with ultra high performance liquid chromatography-tandem mass spectrometry: application for plasma, plasma ultrafiltrate, urine and saliva in a routine laboratory. J Chromatogr B Analyt Technol Biomed Life Sci. 2010;878:2863-9.

23. Sasaki Y, Katabami T, Asai S, Fukuda H, Tanaka Y. In the overnight dexamethasone suppression test, $1.0 \mathrm{mg}$ loading is superior to $0.5 \mathrm{mg}$ loading for diagnosing subclinical Cushing's syndrome based on plasma dexamethasone levels determined using liquid chromatography-tandem mass spectrometry. Endocr J 2017;64:833-42.

24. ICH quality guidelines. Q2 (R1) Validation of Analytical Procedures: Text and Methodology. Available at: https://www.ich.org/page/qualityguidelines. 